\title{
Towards the crystal structure of Channelrhodopsin2
}

Liying Zhang, Kaituo Wang, Pontus Gourdon*

Copenhagen University

The discovery of Channelhodopsins(ChRs) brings a huge breakthrough for optogenetics, especially Channelrhodopsin2's application in neuronal activity. But it is still no known about the molecular mechanism of this channel. The literature describes the structure of C1C2(chimera Channelrhodopsin) at closed state (dark condition) has been solved, but no open state. In fact, even at the blue light condition, there are different states (the circulation between open and closed) during one cycle. It is very difficult to capture the open state. In this thesis, we obtained a mutation of Channelrhodopsin 2 with prolonged open state time so it increases the success rate to obtain the crystals at open state. A mature foreign protein expression system based on S.cerevisiae has been used to express the ChR2 with high expression level. Detergent screens and fluorescence size exclusion has been done and the appropriated detergent has been groped. Lots of setups by different methods have been done and these plates are at the blue light condition since they have been made. Until now we have got some crystals under blue light and these crystals will be sent to Synchrotron. We expect that the crystal structure of ChR2 at open state will be obtained then it will shed much more light about how the channel works during one photocycle and provide much more clues about the precise design of ChR variants to control the neural activity accurately. 